\title{
Guest editorial: soft computing on the web
}

\author{
A. G. López-Herrera · E. Herrera-Viedma
}

Published online: 23 July 2009

(C) Springer-Verlag 2009

This special issue encompasses nine papers devoted to the recent developments in the field of the Soft Computing tools applied on the Web. The issue originated from presentations at the special session "Preference Modelling on the Web" organized by the guest editors at the " 8 th International FLINS Conference on Computational Intelligence in Decision and Control" that was held in Madrid, Spain, 21-24th September 2008. Each paper for the special issue was revised by at least two referees and finally nine papers were accepted according to the referees' evaluations.

The submissions include two papers about the development of information filtering systems or recommender systems based on soft computing tools. The first paper by De Campos, Fernandez-Luna, Huete and Rueda-Morales, "Using second-hand information in collaborative recommender systems", proposes a novel collaborative recommender system that uses quality second-hand information to develop the recommendation process when no collaborative information is available. In the paper "A flexible multi criteria information filtering model", Bordogna and Pasi present a flexible multicriteria information filtering system that allows choosing several distinct criteria, such as content aboutness, coverage, novelty, trust, timeliness, and combining them by a soft aggregation to define a personalized filter in such a way that different personalization levels are possible.

On the other hand, two other papers are related to the discovery of web services. Fenza and Senatore define a system for supporting the user in the discovery of semantic web services, taking into account personal requirements

A. G. López-Herrera · E. Herrera-Viedma ( $\bowtie)$

Department of Computer Science and Artificial Intelligence, University of Granada, 18071 Granada, Spain

e-mail: viedma@decsai.ugr.es and preference in their study entitled "Friendly web services selection exploiting fuzzy formal concept analysis". This approach exploits the fuzzy formal concept analysis for modeling concepts and relative relationships elicited from web resources. In the paper titled "An extension of a fuzzy reputation agent trust model (AFRAS) in the ART testbed", Carbo and Molina-Lopez analyze the performance of an extension of the fuzzy reputation agent trust model (AFRAS) for inferring reability of web services applied in the ART (Agent Reputation and Trust) testbed, showing its advantages and drawbacks.

One contribution of this special issue is related to the development of Web based systems. In the paper titled "Soft computing and web intelligence for supporting consensus reaching", Kacpzryk and Zadrozny introduce a new web-based architecture of a group decision support system for reaching consensus in a group of individuals (agents) based on fuzzy logic. In order to support the consensus reaching process, the authors propose to apply modern web technologies that allow to combine humanconsistency of the assumed representation of the decision problem, intelligent and knowledge-based support for the information search and decision process monitoring and guidance with the user-friendliness and intuitiveness of the user interface, typical for web-based implementations.

In this special issue we give a special attention to the use of the soft computing tools to solve the problem of the information access and information retrieval on the web. Four papers about that topic are included in this issue. In the first one titled "A new fusion strategy for web metasearch", Martín-Bautista, Sáchez, Vila and Delgado present a new rank-based fusion strategy and a score-based fusion strategy with fuzzy scores to solve the collection fusion problem on the web. As novelty, they use RL-preference relations to represent and work with the information, and as a basis for 
the fusion method. The methods provide as output a particular type of preference relation between documents consisting of a ranking of groups of indistinguishable documents, plus a set of incomparable documents. This kind of preference relation is a good tradeoff between accuracy and understandability when showing the result of a fusion process. The second one by Romero, Peralta, Soto, Olivas y Serrano-Guerrero, "Fuzzy optimized self-organizing maps and their application to document clustering", presents an approach using fuzzy logic techniques and self-organizing maps in order to manage conceptual aspects in document clusters and to reduce the training time. In this approach, new fuzzy improvements such as automatic choice of the topology, heuristic map initialization, a fuzzy similarity measure and a keywords extraction process are used to improve the system performance. In the paper "Reasoning about Fuzzy Temporal Information from the Web Towards Retrieval of Historical Events", Schockaert, De Cock and Kerre investigate a framework based on a fuzzyfication of Allen's Interval Algebra to cope with the problem of retrieval of historical events on the web. They use simple heuristic techniques to extract temporal information from web documents and a fuzzy temporal reasoner to improve the reliability of the extracted information, and to deal with conflicts that arise because of the vagueness of events. With such tools a consistent and reliable knowledge base of fuzzy temporal relations can thus be obtained, which effectively allows us to target temporally constrained retrieval tasks. In the paper titled "A quality evaluation methodology for health-related websites based on a 2-tuple fuzzy linguistic approach", Moreno, Morales del Castillo, Porcel and Herrera-Viedma tackle the problem of the web quality evaluation in health-related websites. The authors present a novel qualitative and user-oriented methodology based on the qualitative research tool of focus groups and a 2-tuple fuzzy linguistic approach to evaluate the quality of health-related websites. This methodology means an improvement on quality evaluation of health websites through the commitment to put users first.

We hope that this collection of research papers will contribute to show the usefulness of SC technologies to face the new research challenges related to the Web management, and will stimulate new applications of SC techniques in this context.

Finally, as Guest Editors of this special issue, we would like to thank all the authors for their contributions and the referees for their outstanding cooperation and constructive input. 